\title{
Detecting and studying high-energy neutrinos with FASER $v$ at the LHC
}

\author{
Akitaka Ariga $^{a, *}$ on behalf of the FASER Collaboration \\ a Albert Einstein Center for Fundamental Physics, Laboratory for High Energy Physics, \\ Universität Bern, Sidlerstrasse 5, CH-3012 Bern, Switzerland
}

E-mail: akitaka.ariga@lhep.unibe.ch

FASER, the Forward Search Experiment at the Large Hadron Collider (LHC), is an experiment aiming to search for light, weakly-interacting new particles. The particle detector will be located $480 \mathrm{~m}$ downstream of the ATLAS interaction point. In addition to searches for new particles, we proposed a new detector (FASERv) to study neutrinos at the highest man-made energies and got approval by the CERN Research Board in December 2019. To date, neutrino cross-section data exist up to a few $100 \mathrm{GeV}$ with accelerator-based neutrino beams. With FASERv, the neutrino cross-sections will be measured in the currently unexplored energy range between a few $100 \mathrm{GeV}$ and $6 \mathrm{TeV}$. In particular, electron-neutrino and tau-neutrino cross sections will be measured at the highest energy ever. Furthermore, the channels associated with heavy quark (charm and beauty) production could be studied. As a feasibility study, we performed a test run in 2018 at the proposed detector location with a 30-kg lead/tungsten emulsion detector and collected data of $12.5 \mathrm{fb}^{-1}$. By analyzing the data, we selected several neutrino interaction candidates and are performing a multivariate analysis for the separation from the background towards a first detection of neutrinos at the LHC. From 2022 to 2024 during Run 3 of the LHC, we will deploy an emulsion detector with a target mass of 1.2 tons, possibly coupled with the FASER magnetic spectrometer, which would yield roughly $1,300 v_{e}, 5,800 v_{\mu}$, and $20 v_{\tau}$ interacting in the detector. Here, we present an overview and the status of the FASER $v$ program, as well as the analysis for the neutrino detection in the 2018 data.

40th International Conference on High Energy physics - ICHEP2020

July 28 - August 6, 2020

Prague, Czech Republic (virtual meeting)

\footnotetext{
${ }^{*}$ Speaker
} 


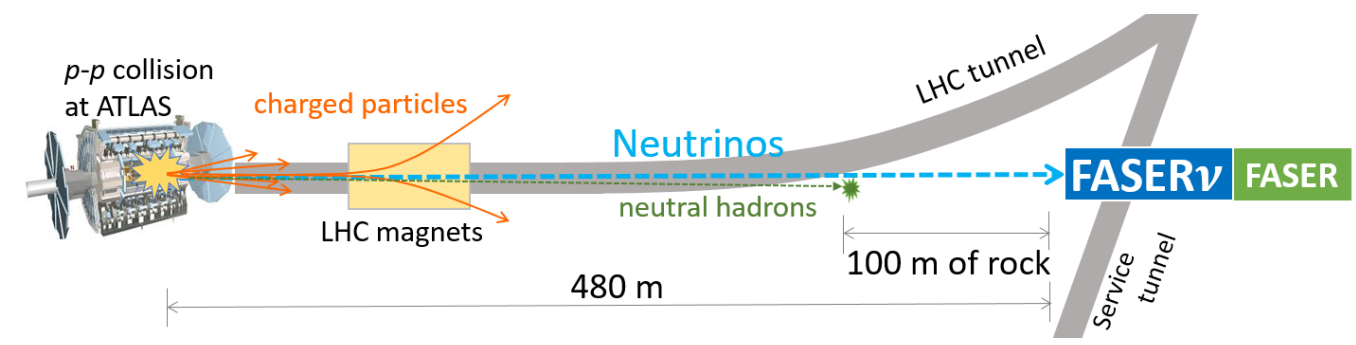

Figure 1: Schematic view of the neutrino beamline for this project.

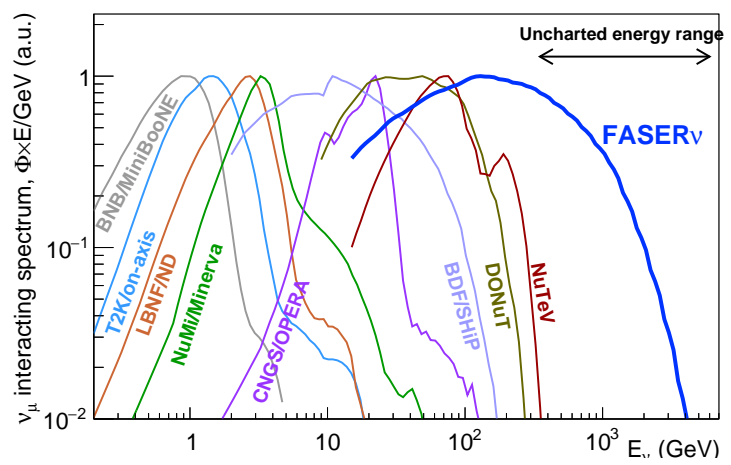

Figure 2: Muon neutrino spectra from different neutrino beamlines. Y-axis is an arbitrarilyscaled interaction rate (number of interactions per $\mathrm{GeV})$.

\section{Neutrinos at the LHC}

The FASER experiment is a newly approved experiment at the CERN-LHC. It has two physics pillars, namely the new long-lived particles search [1] and high-energy neutrino studies (FASER $v$ ) $[2,3]$. This presentation focuses on the neutrino program.

So far, no neutrinos produced at a collider has ever been detected. FASER $v$ aims to detect and study "collider neutrinos" for the first time, by exploiting neutrinos from the LHC. Figure 1 shows the schematics of the neutrino beamline setup proposed for this experiment. An intense forward beam of hadrons is produced at the interaction point in the ATLAS apparatus. A fraction of them decay into neutrinos and leptons. Most of the remaining charged particles are then deflected by the LHC dipole magnets, and neutral hadrons are absorbed in the beamline infrastructure and rock shielding. Neutrinos travel to the detector located $\sim 480 \mathrm{~m}$ downstream of the ATLAS, through about $100 \mathrm{~m}$ of rock. Although not designed for a conventional neutrino beamline, this setup provides essential components of high-energy neutrino beamlines, namely a proton target, a sweeping magnet and a hadron shielding.

By using this beamline, we can exploit neutrinos at the high energy frontier of accelerator neutrinos. Figure 2 shows the comparison of $v_{\mu}$ energy spectra of FASER $v$ and other acceleratorbased experiments/projects. To date, there is an unexplored energy range at the TeV scale between the accelerator data $(E<360 \mathrm{GeV})$ and atmospheric neutrino data $(E>6.3 \mathrm{TeV})$, which will eventually be covered by FASER $v$. Furthermore the beam includes all three neutrino generations, $v_{e}$ and $v_{\tau}$ cross sections will be measured at the highest energy ever. With this beam and a flavorsensitive neutrino detector, we will study production, propagation and interactions of neutrinos at a brand-new kinematical regime. It has implications for neutrino physics, but also for QCD and cosmic ray physics. 

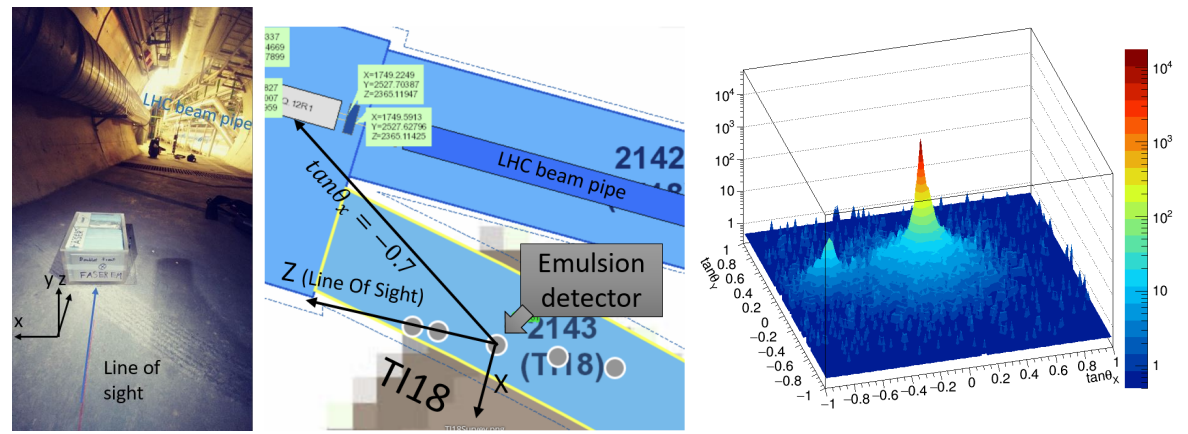

Figure 3: In situ measurements by emulsion detectors at the TI18 location. We show photo of the installed detectors (left), map of the installation location (center), and angular distribution of the detected particle (right).

\begin{tabular}{|c|c|c|}
\hline & normalized flux, all $\left[\mathrm{fb} \mathrm{cm}{ }^{-2}\right]$ & normalized flux, main peak $\left[\mathrm{fb} \mathrm{cm}^{-2}\right]$ \\
\hline TI18 & $(2.6 \pm 0.7) \times 10^{4}$ & $(1.2 \pm 0.4) \times 10^{4}$ \\
\hline TI12 & $(3.0 \pm 0.3) \times 10^{4}$ & $(1.9 \pm 0.2) \times 10^{4}$ \\
\hline \hline \multicolumn{2}{|r|}{ FLUKA (muons, E $>10 \mathrm{GeV})$} & $2 \times 10^{4}$ \\
\hline
\end{tabular}

Table 1: Measured fluxes from emulsion detector data [1]. The fluxes in the main peak (within $10 \mathrm{mrad}$ ) should be compared with the muon flux computed by the FLUKA simulation.

\section{Pilot measurements in $\mathbf{2 0 1 8}$}

In order to prove the feasibility of neutrino measurements, we performed a series of in-situ measurements in 2018. Emulsion detectors were installed in two candidate sites, the so-called TI-18 and TI-12 tunnels, on the beam collision axis. The two candidate sites are located at the symmetric position with respect to ATLAS. The setup and measured angular distribution in the TI-18 tunnel is shown in Figure 3. The main angular peak is consistent with the direction of the $p$ - $p$ collision point. The secondary peak is due to the backward particles from the beamline component. The measured fluxes are reported in Table 1. These particles are found to be mostly muons and electrons, and with a very little hadron component. Muons and electrons do not mimic a neutrino interaction vertex. This result shows that an emulsion detector can work at the actual experimental environment and also neutrino measurements are feasible.

Furthermore, a 30-kg pilot neutrino detector was placed on axis in the TI-18 tunnel, which collected $12.5 \mathrm{fb}^{-1}$ of data. The detector was made of emulsion films interleaved with either 1-mmthick lead plates or 0.5 -mm-thick tungsten plates. Figure 4 reports the setup, reconstructed tracks, and one of the selected neutrino interaction candidates. Although the charged particle density was high, $\simeq 3 \times 10^{5}$ particles $/ \mathrm{cm}^{2}$, the tracks were successfully reconstructed by using the software developed for the NA65/DsTau experiment [4].

An effective target mass of $12.3 \mathrm{~kg}$ has been analyzed, out of which 11 neutral vertices were selected by requesting 5 or more charged particles at the interaction vertex, with their emission angle within $\tan \theta<0.1$ with respect to the beam. The main background is expected from neutral hadrons produced by muons interacting in the upstream rock. Since the lepton identification was not aimed at by the pilot detector, the separation of neutrino and neutral hadron interactions is more 
challenging than that in the physics run. A multivariate method was applied on the topological and kinematical variables. Figure 5 shows the BDT output distributions for the MC and data. The expected number of neutrino signal and neutral hadron background in the given sample are 1.5 and 5.6 events, respectively. A preliminary hypothesis test based on the profile likelihood ratio method was applied on the full range of BDT output distributions, which resulted in a statistical significance of $2.1 \sigma$ away from the background-only hypothesis. This demonstrates the capability to detect neutrinos at the LHC with this detector technology.

\section{Prospects in Run 3 (2022-2024)}

FASER $v$ 's data taking in Run 3 of LHC operation (14 TeV) has been approved by CERN. The FASER apparatus in Run 3 is shown in Figure 6. FASER $v$ detector is a tungsten-emulsion detector with a size of $25 \mathrm{~cm} \times 25 \mathrm{~cm} \times 1.3 \mathrm{~m}$, with a target mass of 1.2 tons. It has a longitudinal length of $10 \lambda$, important for muon identification, and $285 X_{0}$, relevant for electro-magnetic shower reconstruction. FASER $v$ has capabilities to identify lepton flavors and also charm/beauty particles. FASER $v$ is followed by the magnetic spectrometer, which complements muon charge identification. Thanks to this feature, $v_{\mu}$ and $\bar{v}_{\mu}$ will be studied separately. The neutrino energy will be reconstructed in FASER $v$ by combining the topological and kinematical measurable, which would yield
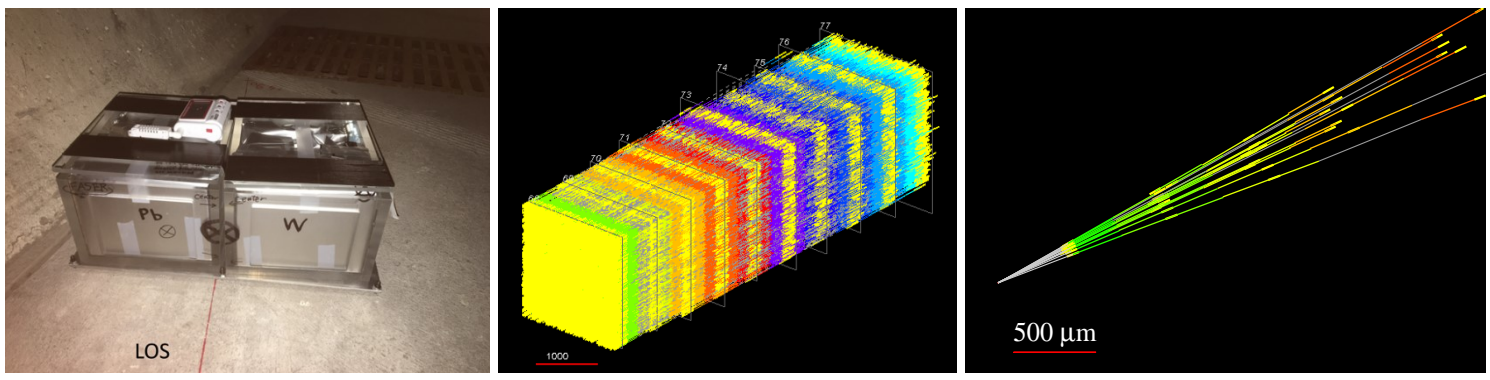

Figure 4: Left: The $30 \mathrm{~kg}$ pilot neutrino detector installed in the LHC tunnel in 2018, which collected 12.5 $\mathrm{fb}^{-1}$ of data. Center: Reconstructed tracks in the $2 \mathrm{~mm} \times 2 \mathrm{~mm} \times 10$ emulsion films. The red scale bar shows $1000 \mu \mathrm{m}$. About 13,000 tracks were observed, corresponding to a track density of $3 \times 10^{5}$ tracks $/ \mathrm{cm}^{2}$. Right: A vertex found in the detector with no incoming charged track. Yellow line segments are the measured tracks in the emulsion films and gray lines are their extrapolations.
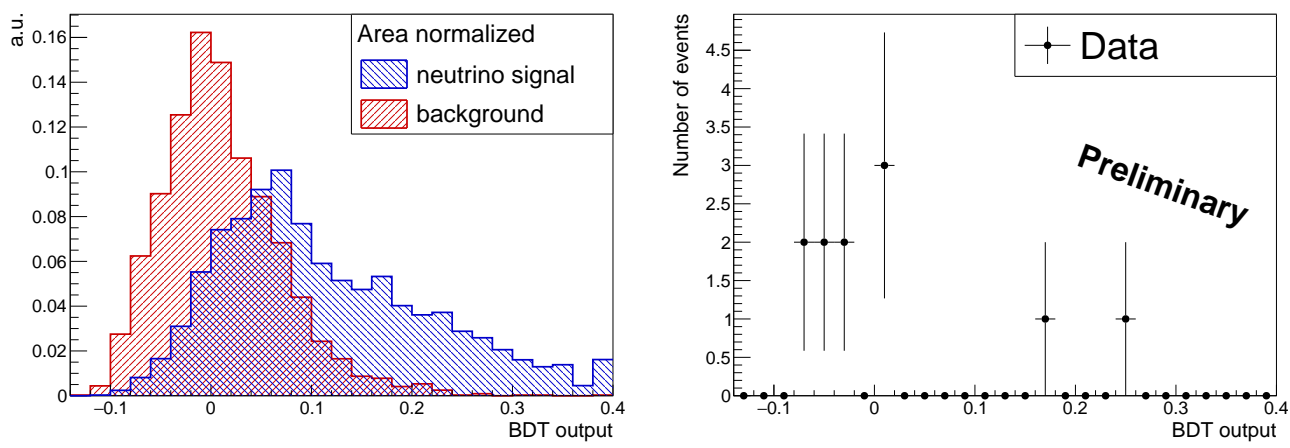

Figure 5: BDT distribution of the MC and data. 


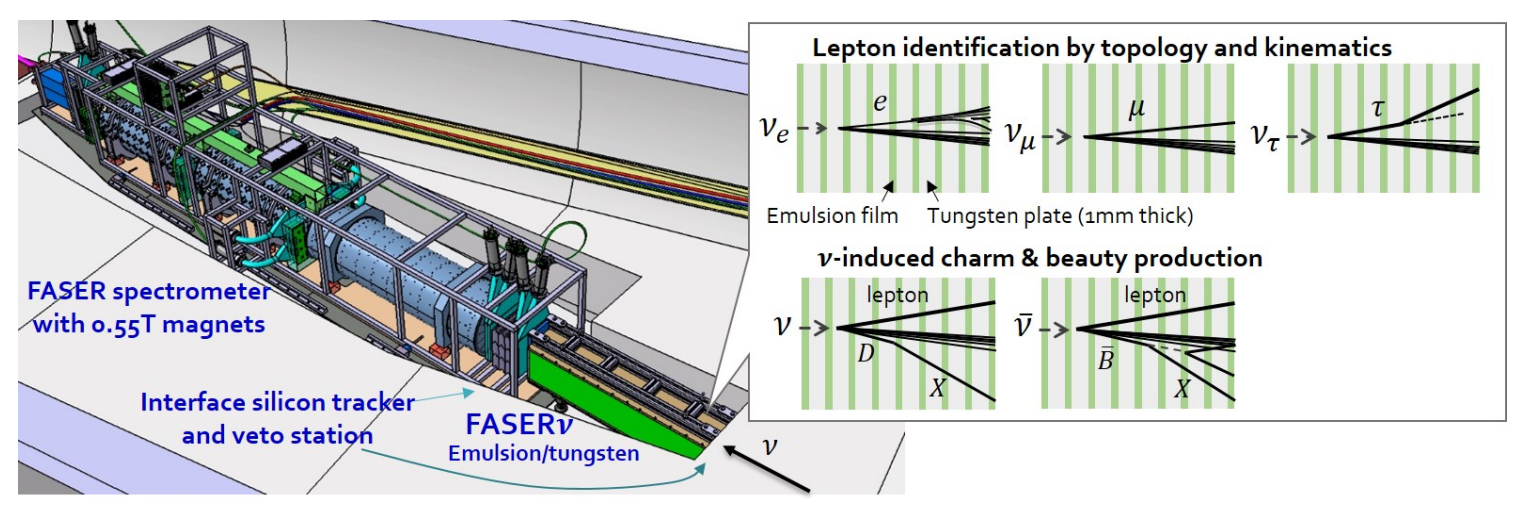

Figure 6: Schematic of the FASER apparatus in Run 3. FASER $v$ will be placed upstream of the spectrometer.

\begin{tabular}{|c|c|c|c|c|}
\hline & FASER estimation & FLUKA-based & Mean interacting energy & Main parent \\
\hline$v_{e}, \bar{v}_{e}$ & 816,447 & 2986,1261 & $830 \mathrm{GeV}$ & $K, D$ \\
\hline$v_{\mu}, \bar{v}_{\mu}$ & 6756,2074 & 8437,2737 & $840 \mathrm{GeV}$ & $\pi, K$ \\
\hline$v_{\tau}, \bar{v}_{\tau}$ & 12,6 & 110,55 & $970 \mathrm{GeV}$ & $D_{s}$ \\
\hline
\end{tabular}

Table 2: Expected number of charged-current interactions occurring in 1.2-ton detector with $150 \mathrm{fb}^{-1}$.

in an energy resolution of about $30 \%$.

During Run 3 with a nominal luminosity of $150 \mathrm{fb}^{-1}$, we expect $O\left(10^{4}\right)$ neutrino interactions occurring in the detector, as detailed in Table 2. Currently, the neutrino flux uncertainty is not negligible. In particular, the forward charm hadron production at the $p$ - $p$ collision has a rather large uncertainty, which affects $v_{e}$ and $v_{\tau}$ interaction rates. In other words, FASER $v$ is sensitive to the QCD problems, namely the gluon/charm PDF in proton or intrinsic charm. These are also an important inputs for astrophysical neutrino observation by neutrino telescopes. The neutrino flux estimation is currently under an intensive review, involving the forward hadron production tuning in Pythia 8 and the beam transportation modeling with the BDSim framework.

Using observed energy-dependent interaction rates and estimated neutrino fluxes, we will study three-flavor neutrino cross sections at the uncharted energy range at $350 \mathrm{GeV}-6 \mathrm{TeV}$. The projected cross section measurement is presented in Figure 7. Furthermore, the charm production rate in charged-current interactions, $R_{c}^{\nu_{\ell}}(E)=N_{\text {charm }}^{\nu_{\ell} \mathrm{CC}} / N_{\text {inclusive }}^{\nu_{\ell} \mathrm{CC}}$, will be compared among three neutrino flavors. This might shed light on the recently-identifed flavor anomalies, which suggests lepton universality violation in heavy meson decays. It is worth noting that FASER $v$ would also constitute the first observation of charm production in $v_{e}$ scattering. There are many more physics subjects, which could be explored by FASER $v$ and are described in [2].

The FASER Collaboration is preparing the detector setup towards the data taking in 2022. As shown in Figure 8, the trench at the detector site has been prepared, which allows placing the FASER $v$ detector on axis. Also the tunnel has been refurbished and ready for the installation of detector components. In parallel, a protection of the LHC beamline has been put in order to prevent a possible damage when transporting the FASER $v$ detector over the beamline. The neutrino beam is expected to be delivered in February 2022. 

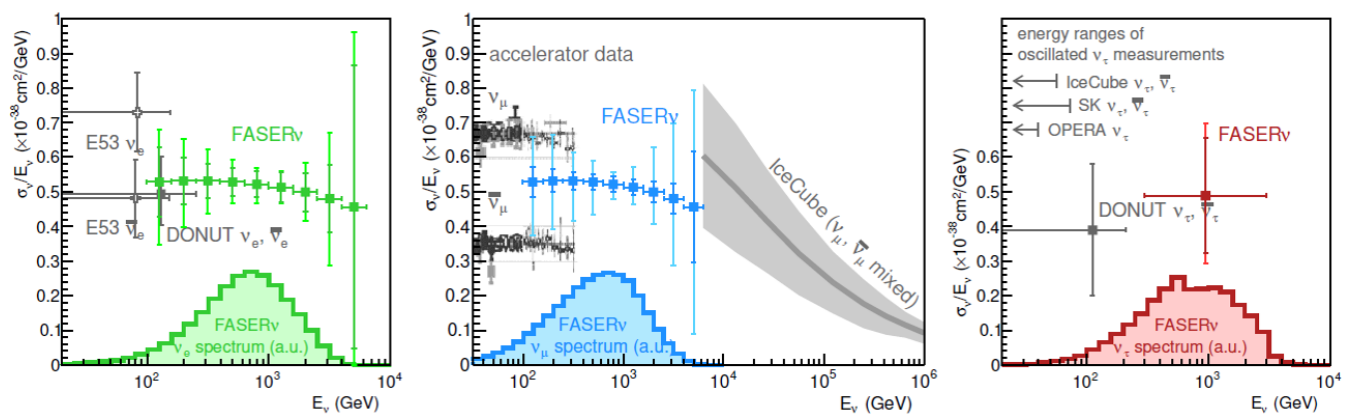

Figure 7: Projected neutrino cross section measurement during Run 3 (2022-2024)
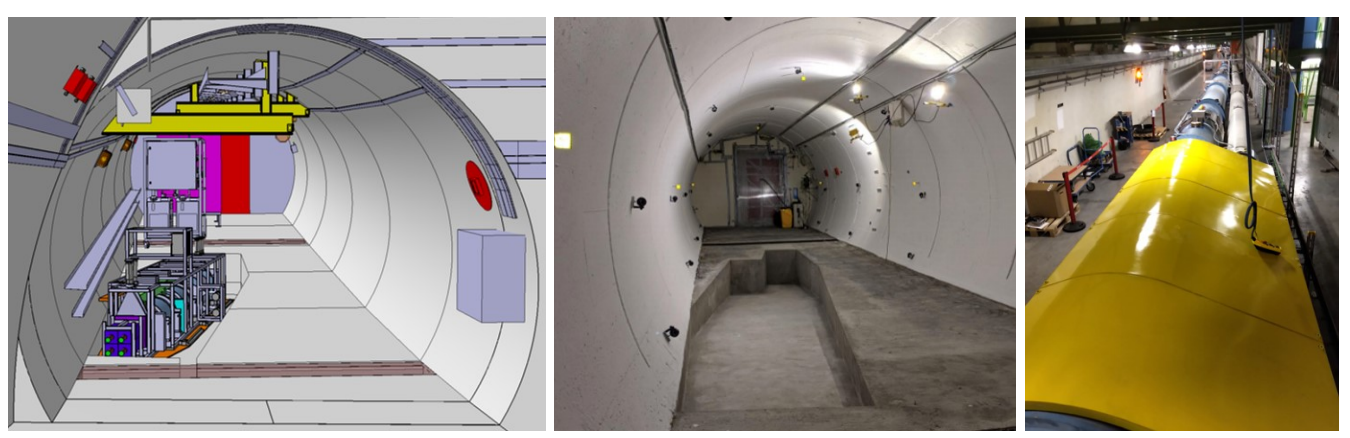

Figure 8: Schematics (left) and photo (center) of the FASER cite in the LHC tunnel, and the protection of the LHC beamline against an accident during the transportation of FASER $v$ detector (right).

\section{Conclusion}

FASER $v$ at the LHC will be the first experiment making use of "Collider neutrinos". It will study neutrinos at the high energy frontier of man-made neutrinos. The in-situ measurements in 2018 quantified the background and also demonstrated the neutrino detection with the proposed method. Data taking in Run 3 (2022-2024) would yield neutrino interaction $O\left(10^{4}\right)$ interactions, which allows sensitive studies of neutrino properties and flavor physics. We are preparing for the physics run in 2022.

\section{References}

[1] FASER collaboration, Technical Proposal for FASER: ForwArd Search ExpeRiment at the LHC, 1812.09139.

[2] FASER collaboration, Detecting and Studying High-Energy Collider Neutrinos with FASER at the LHC, Eur. Phys. J. C80 (2020) 61 [1908. 02310].

[3] H. Abreu, C. Antel, A. Ariga, T. Ariga, J. Boyd, F. Cadoux et al., Technical Proposal: FASERnu, Tech. Rep. CERN-LHCC-2019-017. LHCC-P-015, CERN, Geneva (11, 2019).

[4] DsTau collaboration, DsTau: Study of tau neutrino production with $400 \mathrm{GeV}$ protons from the CERN-SPS, JHEP 01 (2020) 033 [1906. 03487]. 